\title{
Enhanced recovery after surgery and video-assisted thoracic surgery lobectomy: the Italian VATS Group* surgical protocol
}

\author{
Alessandro Gonfiotti ${ }^{1}$, Domenico Viggiano ${ }^{1}$, Luca Voltolini ${ }^{1}$, Alessandro Bertani ${ }^{2}$, Luca Bertolaccini ${ }^{3}$, \\ Roberto Crisci ${ }^{4}$, Andrea Droghetti ${ }^{5}$ \\ ${ }^{1}$ Thoracic Surgery Unit, Careggi University Hospital, Florence, Italy; ${ }^{2}$ Division of Thoracic Surgery and Lung Transplantation, Department for the \\ Treatment and Study of Cardiothoracic Diseases and Cardiothoracic Transplantation, IRCCS ISMETT-UPMC, Palermo, Italy; ${ }^{3}$ Thoracic Surgery \\ Unit, AUSL Bologna, Maggiore-Bellaria Hospital, Bologna, Italy; ${ }^{4}$ Department of Thoracic Surgery, “G. Mazzini” Hospital, University of L'Aquila- \\ Teramo, L'Aquila, Italy; ${ }^{5}$ Thoracic Surgery Unit, ASST Mantova, Mantova, Italy \\ Contributions: (I) Conception and design: A Gonfiotti, D Viggiano; (II) Administrative support: None; (III) Provision of study materials or patients: L \\ Voltolini, A Droghetti, R Crisci; (IV) Collection and assembly of data: L Bertolaccini, A Droghetti; (V) Data analysis and interpretation: A Bertani, L \\ Bertolaccini; (VI) Manuscript writing: All authors; (VII) Final approval of manuscript: All authors. \\ Correspondence to: Alessandro Gonfiotti, MD. Thoracic Surgery Unit, Careggi University Hospital, Largo Brambilla, 1, 50134 Florence, Italy. \\ Email: agonfiotti@alice.it.
}

\begin{abstract}
Enhanced recovery after surgery $\left(\mathrm{ERAS}^{\circledR}\right)$ is a strategy that seeks to reduce patients' perioperative stress response, thereby reducing potential complications, decreasing hospital length of stay and enabling patients to return more quickly to their baseline functional status. The concept was introduced in the late 1990s and was first adopted in patients undergoing open colorectal surgery. Since then, the concept of ERAS has been adopted by multiple surgical specialties. The diffusion of video-assisted thoracic surgery lobectomy (VATS-L) sets also the surgical treatment of lung cancer as a new area for ERAS development. In this paper, we present the Italian VATS Group (www.vatsgroup.org) surgical protocol as part of the ERAS clinical pathway belonging to the VATS-L national database.
\end{abstract}

Keywords: Enhanced recovery after surgery (ERAS); video-assisted thoracic surgery lobectomy (VATS-L); lung cancer

Submitted Dec 28, 2017. Accepted for publication Jan 25, 2018.

doi: $10.21037 /$ jtd.2018.01.157

View this article at: http://dx.doi.org/10.21037/jtd.2018.01.157

\section{Introduction}

Enhanced recovery after surgery (ERAS) is a strategy that seeks to reduce patients' perioperative stress response, thereby reducing potential complications, decreasing hospital length of stay, and enabling patients to return more quickly to their baseline functional status. With the diffusion of video-assisted thoracic surgery lobectomy (VATS-L), the surgical treatment of lung cancer became a new area for the development of ERAS. The ERAS clinical pathway for VATS-L provides also a surgical protocol with clinical recommendations about the specific aspects closely related to the surgical technique and to the management

\footnotetext{
*List of collaborators of the Italian ERAS Group: Jacopo Vannucci, MD (University of Perugia); Antonio D'Andrilli, MD (S. Andrea Hospital, Roma); Majed Refai, MD (Ospedali Riuniti, Ancona); Guendalina Graffigna, MD (Università Cattolica del Sacro Cuore, Milano); Stefano Lovadina, MD (Ospedali Riuniti, Trieste); Marzia Umari (Ospedali Riuniti, Trieste), Paolo Ferrari, MD (IRCCS ISMETT-UPMC, University of Pittsburgh, Palermo); Michele Zuliani, MD (Ospedali Riuniti, Trieste); Marco Taurchini, MD (Casa Sollievo della Sofferenza, San Giovanni Rotondo); Carlo Del Naja, MD (Casa Sollievo della Sofferenza, San Giovanni Rotondo); Domenico Massullo, MD (S. Andrea Hospital, Roma), Olha Putina, MD (ASST Mantova), Nicoletta Pia Ardò (University of Foggia).
} 
Table 1 Recommendations and suggestion for intraoperative management during VATS lobectomy are schematized

\begin{tabular}{ll}
\hline Field of application & Recommendation \\
\hline Access/trocar numbers & VATS-U >> VATS standard (2/3 ports) \\
Parenchymal mobilization & Recommended \\
Pleural tent & Recommended only in selected cases (i.e., severe emphysema) \\
Surgical sealant & Recommended in case of intraoperative air leak \\
Staple-line reinforcement & Recommended only in selected cases (i.e., severe emphysema) \\
"Fissure-less" technique & Not recommended; left to the surgeon's choice \\
Number of pleural drains & Recommended the use of one drainage \\
\hline
\end{tabular}

VATS-U, uniportal VATS; VATS, video-assisted thoracic surgery.

of chest drainage and other invasive devices (1). Following we present the Italian VATS Group (www.vatsgroup.org) surgical protocol as part of the ERAS project belonging to the VATS-L national database.

\section{ERAS and VATS-L}

The surgical aspects closely related to VATS-L to be defined in an ERAS protocol can be identified in the intra and post-operative phase, as it follows:

* Intra-operative phase: technical aspects designed to achieve maximum reduction of trauma and complications;

* Post-operative phase: management and removal criteria for pleural drains and other invasive devices [e.g., nasogastric tube (NGT), central venous catheter (CVC), urinary catheter, arterial catheter].

\section{Intra-operative surgical aspects}

The minimally invasive approach to pulmonary resections is considered the best surgical approach to early stage nonsmall cell lung cancer (NSCLC), showing several advantages over traditional open surgery in terms of postoperative outcomes (2). In order to minimize the surgical trauma and stress during a VATS-L, we identified three fields of action, resumed in Table 1: the minimal invasiveness of VATS (i.e., number of ports/access); the intraoperative strategies for air leak prevention; the number of pleural drainage.

\section{Minimally invasiveness of VATS approach-number of ports and/or thoracic accesses}

The minimal invasiveness of VATS-L is based on the lower impact of its surgical trauma compared to traditional open thoracotomy. The VATS group adopted the universally accepted definition of VATS-L as a procedure without rib spreading, with thoracoscopic vision and a separate dissection of the hilar structures (www.vatsgroup.org). However, several techniques of VATS-L have been described over the years, differing mainly on the number of ports and their location. The number of ports can be discussed as a factor affecting the invasiveness of the surgical procedure and consequently influencing the postoperative functional recovery.

\section{Three- or bi-portal VATS versus mono-portal VATS}

The transition from a standard multi-portal to a mono-portal VATS approach has been reported by several authors as a viable strategy to improve postoperative outcomes, in terms of pain, length of stay and morbidity. This can potentially be translated into a reduction of post-operative hospitalization and faster recovery of patient's daily activities, which is the goal of ERAS program. A recent meta-analysis by Harris et al. (3) compares the outcomes of $n=627$ multi-portal versus $n=1,223$ uni-portal VATS lobectomies for lung cancer from eight observational studies published over the past 2 years; the results showed statistically significant differences in favour of uni-portal VATS in terms of hospital stay $(6.2 \pm 2.6$ vs. $6.7 \pm 3.4$ days, $\mathrm{P}<0.0001)$, chest drain duration $(4.5 \pm 2.2$ vs. $5.4 \pm 2.9$ days, $\mathrm{P}=0.0006)$ and postoperative complications ( $12.0 \%$ vs. $13.7 \%, \mathrm{P}=0.009)$; also postoperative pain was found to be reduced in monoportal procedures but without any statistical significance. To further confirm this data, a recent study by Tamura et al. (4) has shown how single-port technique reduces postoperative pain and increases quality of life in the perioperative period. Again, if we look at the residual pain and paresthesia, Jutley 
et al. (5) and Gonfiotti et al. (6) showed data in favour of mono-portal VATS. Although further randomized studies are needed to validate all the benefits of a mono-portal approach, in our opinion there is sufficient data to assume that also in the field of VATS-L, a lesser surgical trauma on the chest wall may result in a faster functional recovery. In the surgical chapter of our ERAS protocol, we included all the VATS-L techniques described in the VATS Group database (i.e., three-, bi- and mono-portal) but we suggested to shift toward a reduction of port number.

\section{Air leak prevention}

A postoperative air leak is defined by air escaping the lung parenchyma into the pleural space after any kind of surgery in the chest. The literature defines a prolonged air leak (PAL) as an air leak lasting beyond postoperative day 5. PAL is associated with a worse postoperative course, prolonged hospital stay and increased costs. Some authors therefore consider any PAL as a surgical complication. Prevention of a PAL is crucial from an ERAS perspective. In several reports from the Italian VATS group database, the incidence of PAL is reported in up to $7.2 \%$ of patients and this result is consistent with other large series $(7,8)$.

In addition to this evidence, further studies have shown that PAL is associated with an increased risk of postoperative complications. Brunelli et al. (9) reported a higher rate of pleural empyema in patients with PAL; similarly, Varela and colleagues (10) have shown that an air leak beyond the 5 th postoperative day is associated with an increased risk of atelectasis, pneumonia and pleural effusion.

Several surgical strategies have been developed to prevent PAL, mainly adopting two different strategies: reducing the residual pleural space or reinforcing/ protecting the parenchymal suture line. The routinary use of these procedures is not recommended since there isn't a clear evidence of their utility and some of them (i.e., lung sealants) are quite expansive.

The following air leak prevention strategies were taken into account and analysed in our ERAS protocol:

* Pulmonary mobilization: this is an effective strategy for the prevention of PAL, especially after lobar resection. There are several techniques used for this purpose: the simplest and most commonly practiced is the lysis of all pleuro-parenchymal adhesions and pulmonary ligament;

* Pleural tent: creating an apical pleural tent during upper lobectomy or superior bilobectomy is a proven technique to decrease the risk of PAL. The pleural tent is created by removing the parietal pleura from the endothoracic fascia, starting from one of the thoracic access in a circumferential and apical direction. Three randomized trials demonstrated that a pleural tent created at the time of pulmonary resection may significantly decrease the duration of chest drainage and hospitalization (11), reduce the incidence of postoperative air leak (12), decrease air leak and chest drain duration, as well as hospitalization and hospitality costs (13);

* Surgical sealant: in 2010 a review of the Cochrane Database evaluating the use of surgical sealants for the prevention or reduction of postoperative air leak included 16 randomized trials and 1,642 patients (14): six studies showed a statistically significant reduction of air leak duration in the treatment group while three studies showed also a significant reduction in chest drain duration. On the contrary, no study showed a reduction of length of stay. On this basis, the routine use of surgical sealants is nowadays not recommended. However, several studies showed a positive trend in shortening both, chest drain duration and hospital stay, even if without statistical significance (15); on the other hand, most part of these studies do not include the use of a digital drainage system and the policy to remove the drainage based on liquid production, is often quite conservative $(16,17)$. As a consequence, the failure in moving the benefit of the sealant from the air leak duration to the chest drain removal and length of stay, could be the result of a bad chest drain management. We therefore believe that the use of sealants could be useful in a fast-tracking program; we speculate that with the use of digital drainage system and within an ERAS protocol, this bias could be deleted mainly reducing the time discrepancy between the end of the air leak and the removal of chest drain, often linked to a "traditional" post-operative drain management which is far from an "enhanced recovery" philosophy. In our ERAS protocol we suggested the use of sealants in case of intraoperative air leak observed during the inflation test after lung resection has been completed;

* Reinforced staple-line: the use of reinforced staplelines has shown variable results. In patients with severe emphysema undergoing lung resection, several randomized trials suggest the effectiveness of staple line reinforcement in reducing the incidence of air leak, chest drain duration and hospital stay 
Table 2 Recommendations and suggestion for postoperative chest tube management after VATS-L

\begin{tabular}{ll}
\hline Field of application & Recommendation \\
\hline Digital drainage system & Recommended \\
Continuous suction & Recommended for the first $24 \mathrm{~h}$ \\
Drain removal according to fluid production & $<5 \mathrm{~mL} / \mathrm{kg} / 24 \mathrm{~h}$ in case serous liquid \\
Drain removal according to air leak & Recommended after $6 \mathrm{~h}$ of absence and/or $<40 \mathrm{~mL} / \mathrm{min}$ on digital drain system \\
\hline
\end{tabular}

VATS-L, video-assisted thoracic surgery lobectomy.

$(18,19)$; this indication is confirmed also in case of non-anatomic lung resections in patients with a high risk of PAL and/or FEV1 $<60 \%$ of predicted (8). Outside the field of severe emphysema, the utility of reinforced staple line in lung anatomical resections has not been demonstrated (20). In our surgical protocol, we suggested to reinforce the suture line during completion of the fissure only in case of an associated diffuse emphysema;

* Other techniques for post-operative air leak prevention, our protocol also included the following suggestions:

- Minimal dissection of interlobar fissures;

- Avoid the overlap of suture lines;

- Slow closure of the stapling device in thick parenchymal tissue.

Another potentially useful approach is the "fissureless" technique used during VATS-L. Central aspect of this technique is to avoid dissection of incomplete fissures, reducing the risk of parenchymal lesions and consequently of PAL. The fissureless technique has been described for the first time by Temes et al. (21) and then used during VATS-L by Nomori et al. (22) and Loran et al. (23). In a recent best evidence topic in cardiothoracic surgery (24), out of five selected papers, four demonstrated the fissureless technique used in pulmonary lobectomy was superior to conventional lobectomy (CL) in terms of preventing PAL and shortening the time to air leak cessation. The paper concluded that current evidence demonstrates the fissureless technique as significantly better than CL (25). However, even if the fissureless technique is almost universally accepted in upper or middle lobe lobectomies, it has been criticized when used during lower lobectomies, mainly for oncological reasons as it could reduce the effectiveness of VATS lymph node dissection of N1 stations (26). For these reasons we decided to suggest the use of fissureless technique in our ERAS VATS-L protocol, even if we do not consider this choice as mandatory.

\section{Number of pleural drains}

According to fast-tracking, the VATS Group ERAS program suggested using just one pleural drainage $(28 / 30 \mathrm{Fr})$ instead of two after pulmonary lobectomy; placement of the second tube is to be considered when a significant postoperative air leak is predicted or after a bi-lobectomy (27). A single chest drain, by reducing p.o. chest pain, allows an early and easier patient mobilization, which is the goal of the ERAS program $(28,29)$.

\section{Postoperative surgical aspects: chest drain management}

The ideal chest tube management protocol has yet to be determined (30-32). The review of the literature indicates that clinical decisions are often based on institutional practices, physician training, and preferences developed from experience (33). The timing and parameters for chest tubes removal, the need for postoperative suction are still the subject of debate. Optimizing the duration of chest tube drainage after lung resection is crucial for improving quality of care, shortening the hospital stay and reducing costs (10, 34-35).

In developing our ERAS protocol, we discussed three main aspects about chest tube management: (I) deciding whether suction should be applied to chest tubes and its duration; (II) selecting fluid output threshold for the removal of chest tubes; (III) deciding how long after the cessation (observed or digitally recorded) of an air leak the chest tubes should be removed. The chest tube management protocol is resumed in Table 2 .

\section{Chest drain suction application and duration}

The optimal management of suction is a source of continued debate, and thoracic surgeons are usually divided between two different theories: (I) suction applied to chest tubes prolongs air leaks by increasing the amount of air escaping from parenchyma, or (II) suction applied to chest 
tubes decreases the amount of residual space, promoting pleural apposition and healing.

Cerfolio et al. (36) and Marshall et al. (37) supported the first theory that placing chest tubes on water seal is better than suction for reducing air leaks. On the contrary, Brunelli et al. (38) first observed no advantage with the use of the water seal compared with suction in patients undergoing lobectomy, and in 2005 (39) proposed an alternating strategy of less forceful suction $\left(-10 \mathrm{cmH}_{2} \mathrm{O}\right)$ overnight and water seal during the day. This approach after lobectomy seemed to reduce the incidence of PAL, chest tube duration and postoperative hospital stay. Since our scope was not to support one of the two theories, in the ERAS protocol we recommended postoperative suction for the first $24 \mathrm{~h}$, which is widely accepted, avoiding a prolonged $(>24 \mathrm{~h})$ suction maintenance which may be less effective than simple water seal $(40,41)$. Our ERAS protocol indicates the use of a traditional or digitally monitored thoracic drainage system with a set pressure level of $-20 \mathrm{cmH}_{2} \mathrm{O}$ immediately after the operation until the first $24 \mathrm{~h}$ postoperatively.

\section{Liquid production}

The removal of chest tubes based on the production of fluid is controversial since it's based primarily on tradition and dogma more than on clinical studies. Many surgeons use a very conservative range in daily liquid production, such as $150 \mathrm{~cm}^{3} /$ day or lower. Recently, several authors suggested that the removal of chest tubes draining $400-450 \mathrm{~cm}^{3}$ of serous fluid drainage per day is safe while a large consensus statement defined a safe threshold at $300 \mathrm{~cm}^{3} /$ day (40-42). However, the physiology and pathophysiology of the pleural space always refers to a liquid content and a daily replacement which is quantified in $\mathrm{cm}^{3} / \mathrm{kg}$ (43). Several authors pointed out that a cut-off of 3 up to $5 \mathrm{~cm}^{3} / \mathrm{kg}$ of serous liquid appears to be a reasonable threshold it sits within the range of physiological daily pleural fluid filtration and it is suitable for an early chest drain removal without increasing complications and re-admission rates (44).

Based on these clinical evidences, in our ERAS surgical protocol we indicated the removal of chest tube with $5 \mathrm{~cm}^{3} / \mathrm{Kg}$ of fluid drainage/day or less in case of serous pleural liquid.

\section{Air leak}

The absence of an air leak is considered the most important parameter for chest drain removal.

The clinical practice is traditionally to remove chest tubes between 12 and $24 \mathrm{~h}$ after the end of air leaks.
However, this timing is often arbitrary, depending on the observation intervals and on the subjective assessment; moreover, several air leak grading systems are available and used in the clinical practice $(36,45)$.

About observation intervals, it is difficult to plan a time frame able to capture the precise moment of air leak cessation; usually the frequency of observations depends on clinical progress and medical request, with intervals of at least $4 \mathrm{~h}$. As a consequence, the $24 \mathrm{~h}$ of "no observed air leak", may result from a longer period of air leak absence. This bias usually adversely affects the fast track process, delaying chest drain removal and prolonging hospital stay.

Because traditional drainage systems measure and grade air leaks in a subjective manner, interobserver disagreement on the presence of an air leak is frequent (46). The traditional policy allows chest drain removal only after a period of absence of air leak on coughing as assessed by underwater seal. However, an occasional "bubble" can be noted by different surgeons, sometimes leading to a further $24 \mathrm{~h}$ of drain duration.

The introduction in clinical practice of digital drains has allowed to overcome most part of these problems. Using digitally monitored thoracic drainage systems air leakage and pleural pressure can be objectively measured, the presence and rate of air leakage per minute can be seen on a display in real time and the digital data for air leakage and pleural pressure can be retrospectively analyzed (47), confirming the duration in which the air leak has stopped and differentiating between no air-leak and a blocked drain. Another important contribution of digital drainage systems is the paradigm shift that allows the drain to be safely removed in the presence of a continuing (small) air-leak. Furthermore, digital drains comprise a portable system for suction and allow the maintaining of a constant intrapleural negative pressure without limiting an early patient mobilization.

To date, several prospective studies have been published about the real benefits of digital drainages, showing advantages in terms of pulmonary re-expansion, chest drain duration and hospital stay (36-38).

Moreover, with the use of these new devices, the criteria for chest drain removal have been progressively redesigned, and have become more permissive.

Mesa-Guzman et al. demonstrated that a permissive chest digital drain removal protocol allowed the objective removal of drains earlier, reducing hospital stay for patients and costs for the hospital without increasing post-drain removal complications (44). 
Based on these new clinical evidences (48), we adopted permissive criteria for chest drain removal after air leak cessation; the ERAS protocol suggest an interval of $6 \mathrm{~h}$ of no "observed" air leak in case of traditional drains or, if a digital drainage system is used, the threshold of an air leakage less than $20 \mathrm{~mL} / \mathrm{min}$ for more than $6 \mathrm{~h}$.

\section{Acknowledgements}

None.

\section{Footnote}

Conflicts of Interest: The authors have no conflicts of interest to declare.

\section{References}

1. Varadhan KK, Neal KR, Dejong CH, et al. The enhanced recovery after surgery (ERAS) pathway for patients undergoing major elective open colorectal surgery: a meta-analysis of randomized controlled trials. Clin Nutr 2010;29:434-40.

2. Laursen LØ, Petersen RH, Hansen HJ, et al. Video-assisted thoracoscopic surgery lobectomy for lung cancer is associated with a lower 30-day morbidity compared with lobectomy by thoracotomy. Eur J Cardiothorac Surg 2016;49:870-5.

3. Harris CG, James RS, Tian DH, et al. Systematic review and meta-analysis of uniportal versus multiportal videoassisted thoracoscopic lobectomy for lung cancer. Ann Cardiothorac Surg 2016;5:76-84.

4. Tamura M, Shimizu Y, Hashizume Y. Pain following thoracoscopic surgery: retrospective analysis between single-incision and three-port video-assisted thoracoscopic surgery. J Cardiothorac Surg 2013;8:153.

5. Jutley RS, Khalil MW, Rocco G. Uniportal vs standard three-port VATS technique for spontaneous pneumothorax: comparison of post-operative pain and residual paraesthesia. Eur J Cardiothorac Surg 2005;28:43-6.

6. Gonfiotti A, Jaus MO, Barale D, et al. Uniportal videothoracoscopic surgery: our indications and limits. Innovations 2015;10:309-13.

7. Crisci R, Droghetti A, Migliore M, et al. Video-assisted thoracic lobectomy for lung cancer in Italy: the 'VATS Group' Project. Future Oncol 2016;12:9-11.

8. Singhal S, Ferraris VA, Bridges CR, et al. Management of alveolar air leaks after pulmonary resection. Ann Thorac Surg 2010;89:1327-35.
9. Brunelli A, Xiume F, Al Refai M, et al. Air leaks after lobectomy increase the risk of empyema but not of cardiopulmonary complications: a case-matched analysis. Chest 2006;130:1150-6.

10. Varela G, Jimenez MF, Novoa N, et al. Estimating hospital costs attributable to prolonged air leak in pulmonary lobectomy. Eur J Cardiothorac Surg 2005;27:329-33.

11. Okur E, Kir A, Halezeroglu S, et al. Pleural tenting following upper lobectomies or bilobectomies of the lung to prevent residual air space and prolonged air leak. Eur J Cardiothorac Surg 2001;20:1012-5.

12. Allama AM. Pleural tent for decreasing air leak following upper lobectomy: a prospective randomised trial. Eur J Cardiothorac Surg 2010;38:674-8.

13. Brunelli A, Al Refai M, Monteverde M, et al. Pleural tent after upper lobectomy: a randomized study of efficacy and duration of effect. Ann Thorac Surg 2002;74:1958-62.

14. Belda-Sanchís J, Serra-Mitjans M, Iglesias Sentis M, et al. Surgical sealant for preventing air leaks after pulmonary resections in patients with lung cancer. Cochrane Database Syst Rev 2010:CD003051.

15. Gonfiotti A, Santini PF, Jaus M, et al. Safety and effectiveness of a new fibrin pleural air leak sealant: a multicenter, controlled, prospective, parallel-group, randomized clinical trial. Ann Thorac Surg 2011;92:121724; discussion 1224-5.

16. Merritt RE, Singhal S, Shrager JB. Evidence-based suggestions for management of air leaks. Thorac Surg Clin 2010;20:435-48.

17. Malapert G, Hanna HA, Pages PB, et al. Surgical sealant for the prevention of prolonged air leak after lung resection: meta-analysis. Ann Thorac Surg 2010;90:1779-85.

18. Stammberger U, Klepetko W, Stamatis G, et al. Buttressing the staple line in lung volume reduction surgery: a randomized three-center study. Ann Thorac Surg 2000;70:1820-5.

19. Hazelrigg SR, Boley TM, Naunheim KS, et al. Effect of bovine pericardial strips on air leak after stapled pulmonary resection. Ann Thorac Surg 1997;63:1573-5.

20. Miller JI Jr, Landreneau RJ, Wright CE, et al. A comparative study of buttressed versus nonbuttressed staple line in pulmonary resections. Ann Thorac Surg 2001;71:319-22.

21. Temes RT, Willms CD, Endara SA, et al. Fissureless lobectomy. Ann Thorac Surg 1998;65:282-4.

22. Nomori H, Ohtsuka T, Horio H, et al. Thoracoscopic lobectomy for lung cancer with a largely fused fissure. Chest 2003;123:619-22.

23. Loran DB, Woodside KJ, Cerfolio RJ, et al. Predictors of alveolar air leaks. Chest Surg Clin N Am 2002;12:477-88. 
24. Dunning J, Prendergast B, Mackway-Jones K. Towards evidence-based medicine in cardiothoracic surgery: best BETS. Interact Cardiovasc Thorac Surg 2003;2:405-9.

25. Li S, Lv W, Zhou K, et al. Does the fissureless technique decrease the incidence of prolonged air leak after pulmonary lobectomy? Interact Cardiovasc Thorac Surg 2017;25:122-4.

26. Nosotti M, Droghetti A, Luzzi L, et al. First Italian Consensus Conference on VATS lobectomy for NSCLC. Tumori 2017;103:124-35.

27. Bjerregaard LS, Jensen K, Petersen RH, et al. Early chest tube removal after video-assisted thoracic surgery lobectomy with serous fluid production up to $500 \mathrm{ml} /$ day. Eur J Cardiothorac Surg 2014;45:241-6.

28. Miyazaki T, Sakai T, Yamasaki N, et al. Chest tube insertion is one important factor leading to intercostal nerve impairment in thoracic surgery. Gen Thorac Cardiovasc Surg 2014;62:58-63.

29. Zhang X, Lv D, Li M, et al. The single chest tube versus double chest tube application after pulmonary lobectomy: A systematic review and meta-analysis. J Cancer Res Ther 2016;12:C309-16.

30. Deng B, Tan QY, Zhao YP, et al. Suction or non-suction to the underwater seal drains following pulmonary operation: meta-analysis of randomised controlled trials. Eur J Cardiothorac Surg 2010;38:210-5.

31. Coughlin SM, Emmerton-Coughlin HM, Malthaner R. Management of chest tubes after pulmonary resection: a systematic review and meta-analysis. Can J Surg 2012;55:264-70.

32. Lang P, Manickavasagar M, Burdett C, et al. Suction on chest drains following lung resection: evidence and practice are not aligned. Eur J Cardiothorac Surg 2016;49:611-6.

33. Zardo P, Busk H, Kutschka I. Chest tube management: state of the art. Curr Opin Anaesthesiol 2015;28:45-9.

34. Varela G, Jiménez MF, Novoa NM, et al. Postoperative chest tube management: measuring air leak using an electronic device decreases variability in the clinical practice. Eur J Cardiothorac Surg 2009;35:28-31.

35. Cerfolio RJ, Bryant AS. The benefits of continuous and digital air leak assessment after elective pulmonary resection: a prospective study. Ann Thorac Surg 2008;86:396-401.

36. Cerfolio RJ, Bass C, Katholi CR. Prospective randomized trial compares suction versus water seal for air leaks. Ann Thorac Surg 2001;71:1613-7.

37. Marshall MB, Deeb ME, Bleier JI, et al. Suction vs water seal after pulmonary resection: a randomized prospective study. Chest 2002;121:831-5.
38. Brunelli A, Monteverde M, Borri A, et al. Comparison of water seal and suction after pulmonary lobectomy: a prospective, randomized trial. Ann Thorac Surg 2004;77:1932-7.

39. Brunelli A, Sabbatini A, Xiume' F, et al. Alternate suction reduces prolonged air leak after pulmonary lobectomy: a randomized comparison versus water seal. Ann Thorac Surg 2005;80:1052-5.

40. Brunelli A, Beretta E, Cassivi SD, et al. Consensus definitions to promote an evidence-based approach to management of the pleural space. A collaborative proposal by ESTS, AATS, STS, and GTSC. Eur J Cardiothorac Surg 2011;40:291-7.

41. Bardell T, Petsikas D. What keeps postpulmonary resection patients in hospital? Can Respir J 2003;10:86-9.

42. Nomori H, Horio H, Suemasu K. Early removal of chest drainage tubes and oxygen support after a lobectomy for lung cancer facilitates earlier recovery of the 6-minute walking distance. Surg Today 2001;31:395-9.

43. Miserocchi G. Physiology and pathophysiology of pleural fluid turnover. Eur Respir J 1997;10:219-25.

44. Mesa-Guzman M, Periklis P, Niwaz Z, et al. Determining optimal fluid and air leak cut off values for chest drain management in general thoracic surgery. J Thorac Dis 2015;7:2053-7.

45. Macchiarini P, Wain J, Almy S, et al. Experimental and clinical evaluation of a new synthetic, absorbable sealant to reduce air leaks in thoracic operations. J Thorac Cardiovasc Surg 1999;117:751-8.

46. McGuire AL, Petrcich W, Maziak DE, et al. Digital versus analogue pleural drainage phase 1: prospective evaluation of interobserver reliability in the assessment of pulmonary air leaks. Interact Cardiovasc Thorac Surg 2015;21:403-7.

47. Takamochi K, Imashimizu K, Fukui M, et al. Utility of objective chest tube management after pulmonary resection using a digital drainage system. Ann Thorac Surg 2017;104:275-83.

48. George RS, Papagiannopoulos K. Advances in chest drain management in thoracic disease. J Thorac Dis 2016;8:S55-64.

Cite this article as: Gonfiotti A, Viggiano D, Voltolini L, Bertani A, Bertolaccini L, Crisci R, Droghetti A. Enhanced recovery after surgery and video-assisted thoracic surgery lobectomy: the Italian VATS Group* surgical protocol. J Thorac Dis 2018;10(Suppl 4):S564-S570. doi: 10.21037/jtd.2018.01.157 